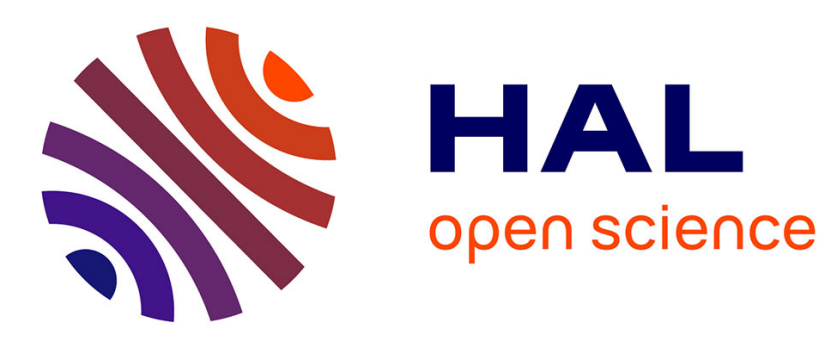

\title{
Maturation-related changes in the development and etiology of neuromuscular fatigue
}

Enzo Piponnier, Vincent Martin, Pierre Bourdier, Brice Biancarelli, Virginie

Kluka, Sebastian Garcia-Vicencio, Anne-Gaelle Jegu, Charlotte Cardenoux, Cedric Morio, Emmanuel Coudeyre, et al.

\section{To cite this version:}

Enzo Piponnier, Vincent Martin, Pierre Bourdier, Brice Biancarelli, Virginie Kluka, et al.. Maturation-related changes in the development and etiology of neuromuscular fatigue. European Journal of Applied Physiology, In press, 119 (11-12), pp.2545-2555. 10.1007/s00421-019-04233-3 . hal-02301647

\section{HAL Id: hal-02301647 \\ https://hal.science/hal-02301647}

Submitted on 30 Sep 2019

HAL is a multi-disciplinary open access archive for the deposit and dissemination of scientific research documents, whether they are published or not. The documents may come from teaching and research institutions in France or abroad, or from public or private research centers.
L'archive ouverte pluridisciplinaire HAL, est destinée au dépôt et à la diffusion de documents scientifiques de niveau recherche, publiés ou non, émanant des établissements d'enseignement et de recherche français ou étrangers, des laboratoires publics ou privés. 


\section{Original article}

2 Maturation-related changes in the development and etiology of 3 neuromuscular fatigue

4 Enzo Piponnier1, Vincent Martin1, Pierre Bourdier1, Brice Biancarelli1, Virginie Kluka1,2, 5 Sebastian Garcia Vicencio1, Anne-Gaëlle Jegu3, Charlotte Cardenoux3, Cédric Morio2, 6 Emmanuel Coudeyre3,4,5, \& Sébastien Ratel 1

7 1: Université Clermont Auvergne, AME2P, F-63000 Clermont-Ferrand, France.

8 2: Decathlon SportsLab, Villeneuve d'Asq, France.

9 3: Clermont University Hospital, Clermont-Ferrand, France.

10 4: Université Clermont Auvergne, UNH, INRA, CRNH Auvergne, F-63000 Clermont-Ferrand, 11 France.

12 Corresponding author:

13 PIPONNIER Enzo

14 Laboratoire AME2P (EA 3533)

15 Campus des Cézeaux

163 rue de la Chébarde

1763178 AUBIERE Cedex

18 Tel: + $33(0) 473405486$

19 Fax: +33(0)4 73407446

20 Email: e.piponnier@yahoo.com 


\section{Abstract}

Purpose: The aim of the present study was to investigate the role of maturation on the etiology of neuromuscular fatigue induced by repeated maximal voluntary isometric contractions (MVIC).

Methods: Nine prepubertal boys $(9.9 \pm 1.3 \mathrm{yr}$.), eight male adolescents $(13.6 \pm 1.3 \mathrm{yr}$.) and eleven men $(23.4 \pm 3.0 \mathrm{yr}$.) performed a series of repeated isometric MVICs of the knee extensors until the MVIC torque reached $60 \%$ of its initial value. Magnetic stimulations were delivered to the femoral nerve every five MVICs to follow the course of voluntary activation level (VA) and the potentiated twitch torque (Qtwpot).

Results: Task failure was reached after $52.9 \pm 12.7,42.6 \pm 12.5$ and $26.6 \pm 6.3$ repetitions in boys, adolescents and men, respectively. VA remained unchanged in men whereas it decreased significantly and similarly in boys and adolescents $(\mathrm{p}<0.001)$. In contrast, Qtwpot remained unchanged in boys and decreased significantly less in adolescents than adults $(\mathrm{p}<0.05)$.

Conclusions: Children and adolescents experience less peripheral and more central fatigue than adults. However, adolescents experience more peripheral fatigue than children for a comparable amount of central fatigue. This finding supports the idea that the tolerance of the central nervous system to peripheral fatigue could increase during maturation.

Keywords: Adolescent, central fatigue, peripheral fatigue, electromyography, peripheral magnetic stimulation

\section{Abbreviations:}

\%CoAct: Level of antagonist co-activation

$\%$ REP: Percentage of the number of repetitions

१2: Partial eta-squared

ANOVA: Analysis of variance

APHV: Age from peak height velocity

BF: Biceps femoris

CI: Confidence interval

EMG: Electromyography

KE: Knee extensors

Mmax: Maximal M-wave amplitude

MVIC: Maximal voluntary isometric contractions

M-wave: Compound action potential

Qtwpot: Potentiated twitch torque

Qtws: Superimposed twitch torque

Qtwunpot: Unpotentiated twitch torque

RF: Rectus femoris

RMS: Root Mean Square

VA: Voluntary activation level

VL: Vastus lateralis 


\section{INTRODUCTION}

Neuromuscular fatigue is commonly defined as "any exercise-induced reduction in the ability of skeletal muscle to produce force or power irrespective of task completion" (Bigland-Ritchie and Woods 1984). Historically, potential factors involved in neuromuscular fatigue were classified into two categories, (i) central factors involving the central nervous system and neural pathways, and (ii) peripheral factors occurring within the muscle, beyond the neuromuscular junction (Gandevia 2001). As a result, it is possible to distinguish both central, i.e. neural, and peripheral, i.e. muscular fatigue. As the neuromuscular system is highly adaptable (Enoka and Stuart 1992), neuromuscular fatigue varies in response to different conditions. For instance, numerous studies examined the differential effect of age (Streckis et al. 2007), ageing (KentBraun et al. 2002), sex (Hunter et al. 2004), training status (Mira et al. 2018) and the mode of contraction (Souron et al. 2018) on neuromuscular fatigue. However, less attention has been paid to the impact of maturation on the development and etiology of neuromuscular fatigue.

Current knowledge suggests that fatigue increases progressively from childhood to adulthood during intermittent whole-body dynamic activities or repeated maximal voluntary contractions. For instance, during repeated cycling sprints, the decline of peak power output is higher in adolescents than children and lower than adults (Ratel et al. 2002). This finding has been confirmed during a series of isokinetic maximal voluntary contractions of the knee extensors (KE) and knee flexors (Zafeiridis et al. 2005; Dipla et al. 2009). Similarly, when muscle contractions include an eccentric phase (i.e. a stretch of the active muscle), the decline of peak torque during exercise is higher in adolescents than children and lower than adults (Chen et al. 2014). Moreover, the magnitude of symptoms appearing during the days following a series of eccentric contractions (i.e. stiffness, oedema, decreased range of motion) is higher in adults than adolescents, and higher in adolescents than children (Chen et al. 2014).

However, it is still unclear whether the contribution of central and peripheral factors to neuromuscular fatigue may differ throughout maturation since no objective comparison of central vs. peripheral fatigue has been performed between prepubertal children, pubertal children (i.e. adolescents) and adults. To date, only Streckis et al. (2007) reported a lower peripheral fatigue and a higher central fatigue during a sustained 2-min maximal voluntary isometric contraction (MVIC) of the KE muscles in 12-14-year old boys and girls than men and women. However, in that study, no objective measurement of maturity status (i.e. biological age) was done, hence limiting our knowledge regarding the role of maturation on the etiology of neuromuscular fatigue. Despite this lack of information, peripheral fatigue could be higher in adolescent individuals than children, but lower in adolescents than adults because of (i) the progressive increase in the strength/power-generating capacity (Tonson et al. 2008) and (ii) potential changes in the metabolic profile throughout maturation, e.g. transformation of slowtwitch fibers into fast-twitch fibers (Lexell et al. 1992) and progressive increase in the capacity of the anaerobic energy turnover over maturation (Ratel and Blazevich 2017). Indeed, it has been shown that the higher neuromuscular fatigue of healthy men $v s$. women was no longer observed when subjects were matched for absolute MVIC force (Hunter et al. 2004) suggesting that the higher the MVIC force, the higher the muscle fatigue. In addition, the amount of peripheral fatigue was found to be greater in individuals with predominantly fast-twitch fibers (Hamada et al. 2003) and relying more on anaerobic than aerobic energy turnover as in explosive power-trained athletes (Garrandes et al. 2007). Taken together, these factors could promote a faster and/or greater development of peripheral fatigue during repeated maximal contractions in adolescents than children but a lower peripheral fatigue in adolescents than adults. Consequently, the development of strength/power and the transformation of slow-twitch into fast-twitch fibers over maturation could be associated with a progressive reduction of time to task failure from childhood to adulthood. As central fatigue is mainly promoted by prolonged 
exercise duration (Thomas et al. 2015), this could translate into a lower central fatigue in adolescents than children as well as in adults than adolescents.

111 Therefore, the purpose of the present study was to verify these assumptions by examining the consequences of maturation on the development and etiology of neuromuscular fatigue induced by repeated voluntary maximal contractions.

\section{MATERIAL AND METHODS}

\section{Participants}

116 A total of nine 8-11 years old boys (Tanner stage 1-2), eight 13-16 years old male adolescents 117 (Tanner stage 3-4) and eleven 18-25 years old healthy men were recruited. All the participants 118 were involved in different physical activities such as rugby, basketball, swimming, etc. To be included, participants had to exercise less than 4 hours per week and to be free of any medical contra-indication to physical activity. The present study was approved by the local Ethics Committee (Protection Committee of People for Biomedical Research South East VI; Authorization Number AU929). All the participants were fully informed of the experimental procedures and gave their written consent before any testing was conducted. In addition, the written consent of the parents/guardians was also obtained for the children and adolescents.

\section{Experimental procedure (design)}

All participants were tested on three experimental sessions separated by at least 1 week. The first session was dedicated to collecting participants' physical characteristics, familiarization with the experimental procedures and clinical examination by a medical practitioner or a pediatrician. During the second session, the participants were asked to perform MVIC of the $\mathrm{KE}$ muscles at different knee angles $\left(30,40,50,60,70,75,80,85,90,100,110^{\circ} ; 0^{\circ}=\right.$ full extension) in a randomized order to determine the optimal angle for maximal torque production. Participants performed two MVICs at each knee angle. Finally, during the third session, all the participants performed the intermittent voluntary fatigue protocol at the optimal knee angle. All sessions were done in a temperate room $\left(18-22^{\circ} \mathrm{C}\right)$.

\section{Anthropometric measurements}

Body mass was measured to the nearest $0.1 \mathrm{~kg}$ using a calibrated scale and height was determined to the nearest $0.01 \mathrm{~m}$ using a standing stadiometer. Height and body mass were measured without shoes and while wearing underwear only. Sitting height was also measured while the participants sat on the floor against a wall, using the same stadiometer. Body mass index was calculated using a standard formula, mass divided by height squared $(\mathrm{kg} \cdot \mathrm{m}-2)$. Body fat and fat-free mass were determined using dual-energy X-ray absorptiometry (HOLOGIC, QDR-4500, Hologic Inc, Bedford, Massachusetts, USA).

\section{Maturity status assessment}

Two methods were used to assess children and adolescents' maturity status: 1) Tanner stages were determined from self-reported assessment based on pubic hair and testicular/penis development (Tanner and Whitehouse 1976), the children and adolescents being assisted by their parents while completing the questionnaire; 2) Age from peak height velocity (APHV) was used to assess somatic maturity and determined by using height, sitting height and body mass (Mirwald et al. 2002).

\section{Intermittent voluntary fatigue protocol}

Participants performed an intermittent voluntary fatigue protocol consisting of a repetition of isometric 5-s MVICs of the KE muscles interspersed with 5-s passive recovery periods until the voluntary torque failed to reach a target value of $60 \%$ of its initial value over three consecutive MVICs. The participants had no visual feedback of torque output during the 
exercise. However, they were strongly encouraged by the investigators during the entire fatiguing task. During the fatigue protocol, all participants were allowed to drink water ad libitum. The knee joint was fixed at the optimal angle for maximal torque production $(75.6 \pm$ $5.3^{\circ}, 90.0 \pm 0.0^{\circ}$ and $81.8 \pm 7.5^{\circ}$ in boys, adolescents and men, respectively; $\left.\mathrm{p}<0.001\right)$, which was determined from the torque-angle relationship during the second visit. The number of repetitions was considered as the performance criteria to quantify neuromuscular fatigue. Single magnetic stimulations were delivered to the femoral nerve every five MVICs to determine the maximal level of voluntary activation (VA) by using the twitch interpolation technique (see below for further details). The electromyographic (EMG) activity of the vastus lateralis (VL) and rectus femoris (RF) muscles was recorded during the entire fatigue protocol. The amplitude of the potentiated twitch torque (Qtwpot; see below) and VL and RF concomitant compound action potential amplitudes (maximal $\mathrm{M}$-wave; $\mathrm{M} \max$ ) were considered as indicators of peripheral fatigue. The time-course of VA and normalized EMG (see below) of the VL and RF muscles throughout the protocol were considered as indexes of central fatigue. Examples of raw data (MVIC torque and VL EMG; panel A) and time-courses of MVIC torque, Qtwpot and VA during the fatigue protocol in a typical boy, adolescent and man are presented in Figure 1.

a
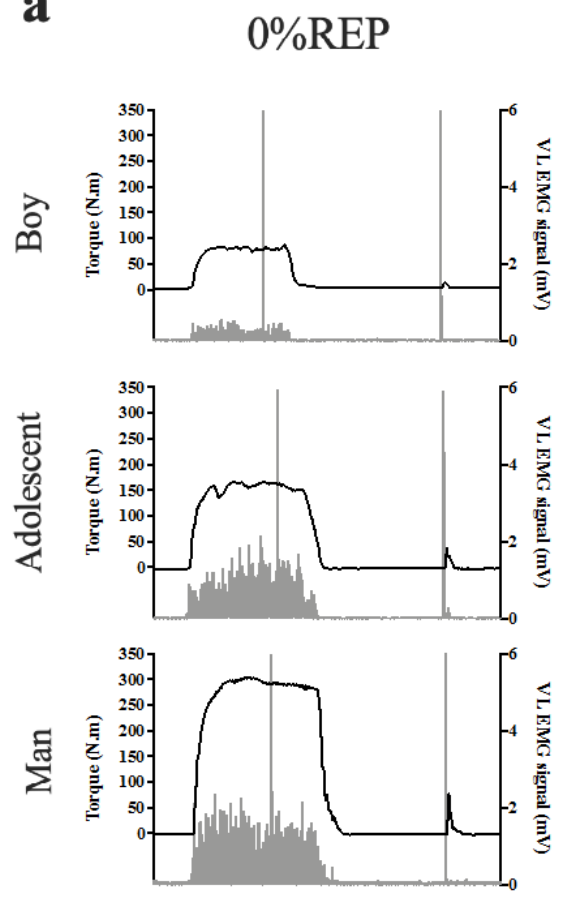
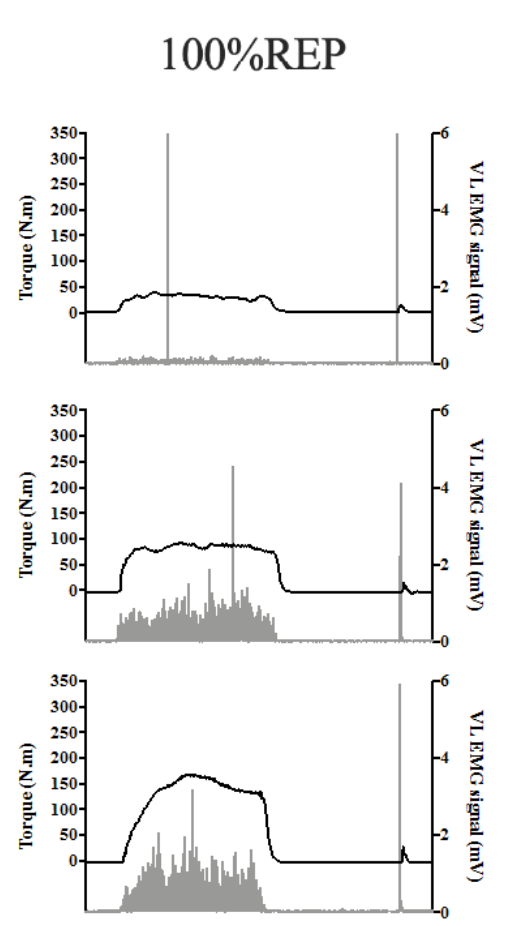

b
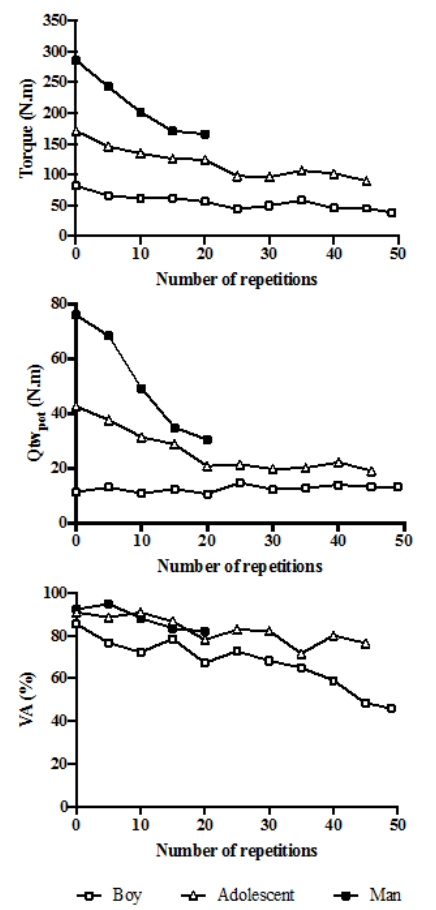

Fig. 1: (a) Example of raw data for MVIC torque and VL EMG obtained at 0\%REP and 100\%REP in a typical boy (top), adolescent (middle), and man (bottom). (b) Example of timecourses of MVIC torque (top), Qtw pot (middle), and VA (bottom) during the intermittent fatigue protocol in a typical boy, adolescent and man.

\section{Torque measurements}

Voluntary and evoked contractions were assessed in isometric condition with an isokinetic dynamometer (Cybex Norm, Lumex, Ronkonkoma, NY, USA). Participants were comfortably positioned on an adjustable chair with the hip joint flexed at $30^{\circ}\left(0^{\circ}=\right.$ neutral position $)$. The dynamometer lever arm was attached $1-2 \mathrm{~cm}$ above the lateral malleolus with a Velcro strap. The axis of rotation of the dynamometer was aligned with the lateral femoral condyle of the right femur. Torque data were corrected for gravity using the Cybex software and were digitized 
to an external A/D converter (Powerlab 8/35, ADInstruments, New South Wales, Australia) driven by the Labchart 7.3 Pro software (ADInstruments, Australia). Femoral nerve stimulation

Evoked contractions of the KE muscles were triggered by a single magnetic stimulus, delivered to the femoral nerve using a 45-mm figure-of-eight coil connected to a magnetic stimulator (Magstim 2002, peak magnetic field strength $2.34 \mathrm{~T}$, stimulation duration $0.1 \mathrm{~ms}$; MagstimCo, Whiteland, Dyfed, UK). The coil was placed high in the femoral triangle in regard of the femoral nerve. Small spatial adjustments were initially performed to determine the optimal position where the greatest unpotentiated KE twitch amplitude (Qtwunpot) and the greatest VL and RF Mmax were evoked. The optimal stimulation intensity, i.e. the intensity where maximal twitch and concomitant $\mathrm{M}$-waves amplitudes started to plateau, was determined from a recruitment curve. Qtwunpot and $\mathrm{M}_{\max }$ plateaued at $85.0 \pm 4.3 \%, 84.4 \pm 7.8 \%$ and $85.0 \pm 6.7 \%$ of the stimulator power output for prepubertal boys, adolescents and men, respectively. In order to overcome the potential confounding effect of axonal hyperpolarization (Burke 2002), the

\section{EMG recordings}

stimulation intensity was set to $100 \%$ of the stimulator output during the subsequent testing procedures (i.e. voluntary intermittent fatigue protocol). This intensity corresponded to $117.9 \pm$ $6.0 \%, 119.5 \pm 11.7 \%$ and $118.4 \pm 10.1 \%$ of the optimal intensity in the boys, adolescents and men, respectively, and was not significantly different between groups. This supramaximal intensity of stimulation $(\approx 120 \%$ ) has been demonstrated as being optimal for an adequate assessment of central and peripheral fatigue of the KE muscles (Neyroud et al. 2014).

\section{Voluntary activation level}

To determine VA, the twitch interpolation was used. Briefly, a superimposed (Qtws) and a potentiated (Qtwpot) single twitch were delivered during MVIC after the torque had reached a plateau, and 3-s after the cessation of the contraction, respectively. These superimposed and potentiated mechanical amplitudes allowed the quantification of VA (\%VA) as proposed by Merton (Merton 1954) (Equation 1):

\section{(Equation 1)}

The EMG signals of the VL, RF and biceps femoris (BF) muscles were recorded using bipolar silver chloride surface electrodes (Blue Sensor N-00-S, 30 x 22 mm, Ambu, Denmark) during voluntary and evoked contractions. The recording electrodes were taped lengthwise on the skin over the muscle belly, as recommended by SENIAM (Surface ElectroMyoGraphy for the NonInvasive Assessment of Muscles) (Hermens et al. 2000) with an inter-electrode distance of 20 $\mathrm{mm}$. The reference electrode was attached to the patella. Low impedance $(\mathrm{Z}<5 \mathrm{k} \Omega)$ at the skinelectrode surface was obtained by shaving, gently abrading the skin with thin sand paper and cleaning with alcohol. EMG signals were amplified (Dual Bio Amp ML 135, ADInstruments, Australia) with a bandwidth frequency ranging from $10 \mathrm{~Hz}$ to $500 \mathrm{~Hz}$ (common mode rejection ratio $>85 \mathrm{~dB}$, gain $=1,000)$ and simultaneously digitized together with the torque signals $(2$ $\mathrm{kHz}$ ). During the course of the fatigue protocol, Root Mean Square (RMS) values of the VL and RF EMG activity were calculated during the MVIC trials over a 0.5 -s period before the superimposed stimulation. This RMS value was then normalized to the maximal peak-to-peak amplitude of the potentiated VL and RF M-waves (RMS.Mmax-1).

\section{Antagonist co-activation}

The level of antagonist co-activation (\% CoActBF) of the BF muscle was computed as the BF EMG activity during knee extensions, normalized to the maximal BF EMG activity recorded during a maximal knee flexion at the optimal angle for maximal KE torque production (Equation 2). To record this maximal BF RMS value, the participants were asked to perform 3- 
s MVICs of the knee flexors before the fatigue protocol. This measurement was repeated twice, and the best trial was used for subsequent analysis.

$$
\% \text { CoAct }=\frac{R M S_{\text {anta }}}{R M S_{\text {ago }}} \cdot 100
$$

\section{(Equation 2)}

Where $\mathrm{RMS}_{\text {anta }}$ is the RMS value of BF during intermittent contractions, and $\mathrm{RMS}_{\mathrm{ago}}$ is the RMS value of BF during maximal voluntary knee flexion, recorded before the fatigue protocol.

\section{Statistical analysis}

The participants were categorized according to their age and maturity level. There were three groups (prepubertal boys, adolescents and men). Therefore, we considered the group as an independent categorical variable while the other variables (i.e. neuromuscular parameters) were dependent variables.

All variables measured during the intermittent fatigue protocols were linearly interpolated between the nearest values at $20 \%, 40 \%, 60 \%$, and $80 \%$ of number of repetitions (\%REP) to fairly compare the different groups. Values at $0 \%$ REP and $100 \%$ REP corresponded to pre- and post-fatigue values, respectively.

Data were screened for normality of distribution and homogeneity of variances using a ShapiroWilk normality test and the Barlett's test, respectively. All the data were normally distributed, and variances were homogeneous. One-way analysis of variance (ANOVA) were used to compare age and anthropometric characteristics between groups. When ANOVA revealed significant effects, Tukey HSD post hoc tests were applied to test the discrimination between groups. Differences in absolute values were analyzed using a two-way (group $\times \%$ REP) ANOVA with repeated measures. When significant differences in initial values were identified between groups, two-way (group $\times \%$ REP) ANOVA were applied to test differences in percent changes relative to the pre-fatigue values. The effect size and statistical power were reported when significant main or interaction effects were detected. When the ANOVA revealed significant effects or interactions between factors, a Tukey HSD post hoc test was applied to test the discrimination between means. The effect size was assessed using the partial etasquared $\left(\eta_{2}\right)$ and ranked as follows: $\sim 0.01=$ small effect, $\sim 0.06=$ moderate effect, and $\geq 0.14$ $=$ large effect (Cohen 1969).

Moreover, to discriminate the effect of MVIC torque on the etiology of neuromuscular fatigue, we used a mixed general linear model: the initial MVIC torque was used as a continuous predictor variable (= co-variable), the group as a categorical independent variable and the Qtwpot or VA as dependent variables. Pearson's correlation coefficients were used to determine linear correlations between the initial MVIC torque, the total number of repetitions and Qtwpot and VA variations over the fatigue protocol. The limit for statistical significance was set at $\mathrm{p}<0.05$. Statistical procedures were performed using the Statistica 12.0 software (Statsoft, Inc, USA).

\section{RESULTS}

\section{Participants' characteristics}

Participants' characteristics are described in Table 1. The boys were prepubertal (Tanner stages I and II and far from their peak height velocity: $-3.8 \pm 0.7 \mathrm{yr}$.) while the adolescents were circapubertal (Tanner stages III and IV and around their peak height velocity: $-1.1 \pm 1.3 \mathrm{yr}$.).

\section{Number of repetitions}

Task failure, corresponding to the predetermined 60\% decrement of MVIC, was reached after $52.9 \pm 12.7$ [CI 95\% = 43.1-62.6], $42.6 \pm 12.5$ [CI 95\% $=32.2-53.0]$ and $26.6 \pm 6.3$ [CI 95\% $=$ 
22.4-30.9] repetitions in boys, adolescents and men, respectively. ANOVA revealed a significant group effect for the total number of repetitions $\left[F(2 ; 25)=15.80, p<0.001, \eta_{2}=0.56\right.$, power $=1.0]$. The total number of repetitions was significantly higher in boys and adolescents than in men $(\mathrm{p}<0.001)$. The number of repetitions tended to be higher in boys than adolescents $\left(\mathrm{p}=0.056, \eta_{2}=0.81\right.$, power $\left.=0.39\right)$.

\section{MVIC torque}

279 Significant interactions (group $\times \%$ REP) were found for the absolute MVIC torque $[\mathrm{F}(10 ; 125)$ $280=14.78, \mathrm{p}<0.001, \eta^{2}=0.55$, power $\left.=1.0\right]$. As expected, initial MVIC torque values were significantly lower in boys than adolescents and men, and in adolescents than men $(87.4 \pm 31.2$ [CI 95\% = 63.4-111.3], $184.2 \pm 47.9$ [CI 95\% = 144.2-224.3] and 298.4 \pm 50.5 [CI 95\% = 264.4-332.3] N.m in boys, adolescents and men, respectively; $\mathrm{p}<0.001)$.

A \%REP effect was found for MVIC torque changes, i.e. in percentage of initial values $\left[\mathrm{F}(4 ; 100)=89.96, \mathrm{p}<0.001, \eta^{2}=0.78\right.$, power $\left.=1.0\right]$. MVIC torque significantly and progressively decreased throughout the fatigue test regardless of group (Fig. 2a).

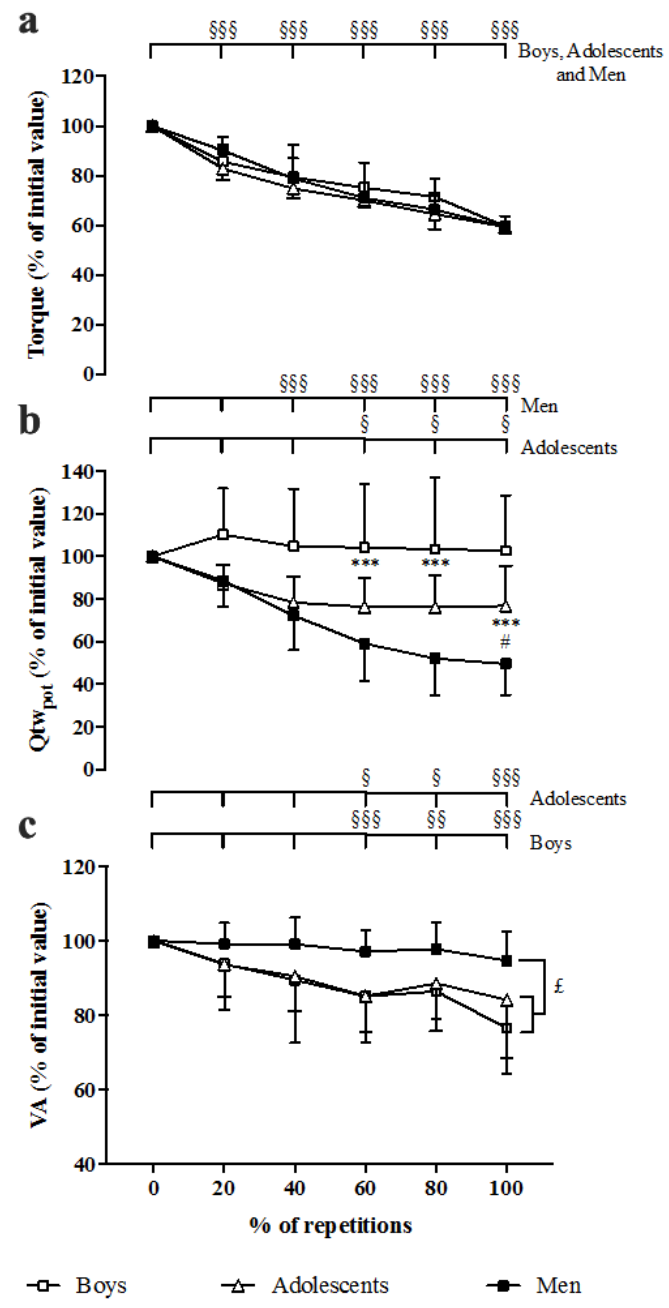

Fig 2: Time-courses of (a) MVIC torque of the knee extensor muscles, (b) potentiated twitch torque (Qtwpot) and (c) voluntary activation level (VA), expressed as a percentage of the initial values, during the fatigue protocol in prepubertal boys, male adolescents and men. ***: Significantly different between boys and men at $\mathrm{p}<0.001$ (identified from statistical analysis on the relative values); \#: Significantly different between adolescents and men at $\mathrm{p}<0.05$ (identified from statistical analysis on the relative values); $£$ : Significantly different over the 
fatigue protocol at $\mathrm{p}<0.05$ (identified from statistical analysis on the absolute values);" $\S, \S \S$ and $\S \S \S$ significantly different from the initial value at $\mathrm{p}<0.05, \mathrm{p}<0.01$ and $\mathrm{p}<0.001$, respectively (identified from statistical analysis on the absolute values). Potentiated twitch torque

During the fatigue protocol, ANOVA revealed a significant interaction of group $\times \%$ REP for absolute Qtwpot values $\left[F(10 ; 125)=16.72, p<0.001, \eta^{2}=0.57\right.$, power $\left.=1.0\right]$. Initial absolute Qtwpot values tended to be lower in boys than in adolescents $(\mathrm{p}=0.07)$ and were lower in boys than in men $(23.2 \pm 9.3$ [CI 95\% $=16.0-30.3$ ], $56.8 \pm 18.9$ [CI 95\% $=38.8-71.3$ ] and $79.3 \pm$ 28.0 [CI 95\% $=60.5-98.0]$ N.m in boys, adolescents and men, respectively; $\mathrm{p}<0.001)$. No significant difference was found between adolescents and men for initial Qtwpot values. Adolescents and men showed a significant decrement of Qtwpot during the fatigue exercise, while it remained unchanged in boys (Fig. 2b).

Furthermore, a significant interaction of group $\times \%$ REP was observed for relative Qtwpot, i.e. in percentage of initial values $\left[\mathrm{F}(8 ; 100)=8.19, \mathrm{p}<0.001, \eta^{2}=0.40\right.$, power $\left.=0.99\right]$. The Qtwpot decreased respectively up to $20 \%$ and $60 \%$ REP and then remained unchanged until the end of the fatigue protocol in adolescents and men. At the end of the fatigue protocol, men showed a greater relative decrement of Qtwpot than adolescents $(-50.5 \pm 14.5 \%$ and $-23.2 \pm 18.9 \%$, respectively; Fig. 2b).

When the initial MVIC torque value was used as co-variable, a significant interaction of group $\times \%$ REP on the course of Qtwpot was observed $(\mathrm{p}<0.05)$. Post-hoc analyses revealed that the decrement of Qtwpot was higher in men than adolescents $(\mathrm{p}<0.01)$ and higher in adolescents than boys $(\mathrm{p}<0.05)$.

\section{Voluntary activation level}

ANOVA revealed a significant interaction of group $\times \%$ REP for absolute VA values $[\mathrm{F}(10 ; 125)$ $=2.20, \mathrm{p}<0.05, \eta^{2}=0.15$, power $\left.=0.90\right]$. Before the fatigue test, the VA values were not significantly different between groups $(87.1 \pm 7.6 \%$ [CI 95\% $=81.2-92.9], 84.4 \pm 5.5 \%$ [CI $95 \%=75.5-91.8], 92.2 \pm 3.2 \%$ [CI 95\% $=90.0-94.3]$ in boys, adolescents and men, respectively). Boys and adolescents showed a significant decrement in VA during the fatigue protocol, whereas it remained unchanged in men (Fig. 2c). At the end of the fatigue test, VA decrements were $-23.4 \pm 12.2 \%$ and $-15.8 \pm 15.7 \%$ in boys and adolescents, respectively.

However, when the MVIC torque was used as co-variable, no significant interaction or main effect on the time-course of VA was observed.

\section{EMG activity}

No significant main or interaction effect was found for the VL and RF Mmax values.

However, there were significant interaction (group $\times \%$ REP) effects for VL and RF absolute RMS.M $M_{\max -1}\left[\mathrm{~F}(10 ; 125)=2.01, \mathrm{p}<0.05, \eta^{2}=0.14\right.$, power $=0.86$ and $\mathrm{F}(10 ; 125)=2.56$, $\mathrm{p}<0.01, \eta_{2}=0.18$, power $=0.94$, respectively]. No difference was found for the initial values of VL and RF RMS.Mmax-1 between age groups. Post hoc tests only revealed a decrement of VL and RF RMS.Mmax-1 in boys (at least $\mathrm{p}<0.01$ ).

\section{Antagonist co-activation}

ANOVA revealed a significant interaction (group $\times \%$ REP) regarding absolute \%CoAct $\left[\mathrm{F}(10 ; 125)=2.72, \mathrm{p}<0.01, \eta^{2}=0.18\right.$, power $\left.=0.96\right]$. No difference was found for the initial value of \%CoAct between age groups. \%CoAct values remained unchanged in adolescents and men during the entire fatigue protocol whereas in boys, it decreased significantly at $60 \%$ REP, 80\% REP and $100 \%$ REP compared to $0 \%$ REP (Fig. 3). 


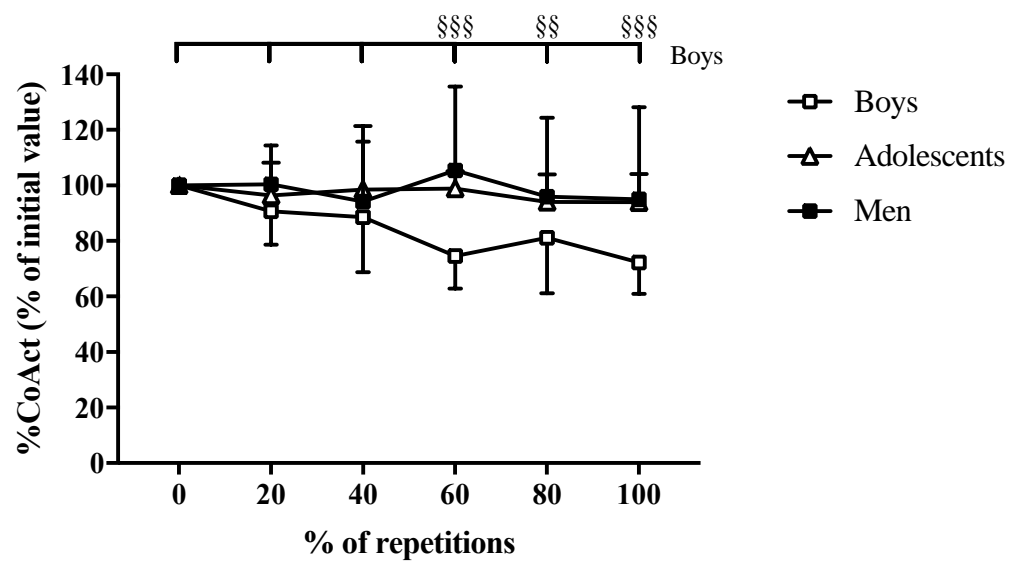

338 Fig. 3: Time-course of the coactivation level of the biceps femoris (\%CoAct) during the fatigue 339 protocol in boys, adolescents and men. $\S \S$ and $\S \S \S$ : significantly different from the initial value 340 at $\mathrm{p}<0.01$ and $\mathrm{p}<0.001$, respectively (identified from statistical analysis on the absolute values).

\section{Correlations}

342 When the three groups were pooled in the analysis, the first MVIC torque of the fatigue protocol 343 was correlated to the number of repetitions $\left(\mathrm{r}_{2}=0.59, \mathrm{p}<0.001\right)$ and relative Qtwpot changes 344 ( $\mathrm{r} 2=0.52, p<0.001)$ (Fig. 4); the higher the MVIC torque, the lower the number of repetitions 345 and the greater the decrement of Qtwpot. In contrast, a significant positive relationship was found 346 between the first MVIC torque and the relative VA decrement $\left(\mathrm{r}_{2}=0.41, p<0.001\right)$; the higher the MVIC torque, the lesser the decrement of VA. Furthermore, the number of repetitions was correlated to the relative VA decrement $\left(\mathrm{r}_{2}=0.23, \mathrm{p}<0.05\right)$ and to the relative Qtwpot decrement $349 \quad(\mathrm{r} 2=0.33, \mathrm{p}<0.01)$ (Fig. 4).
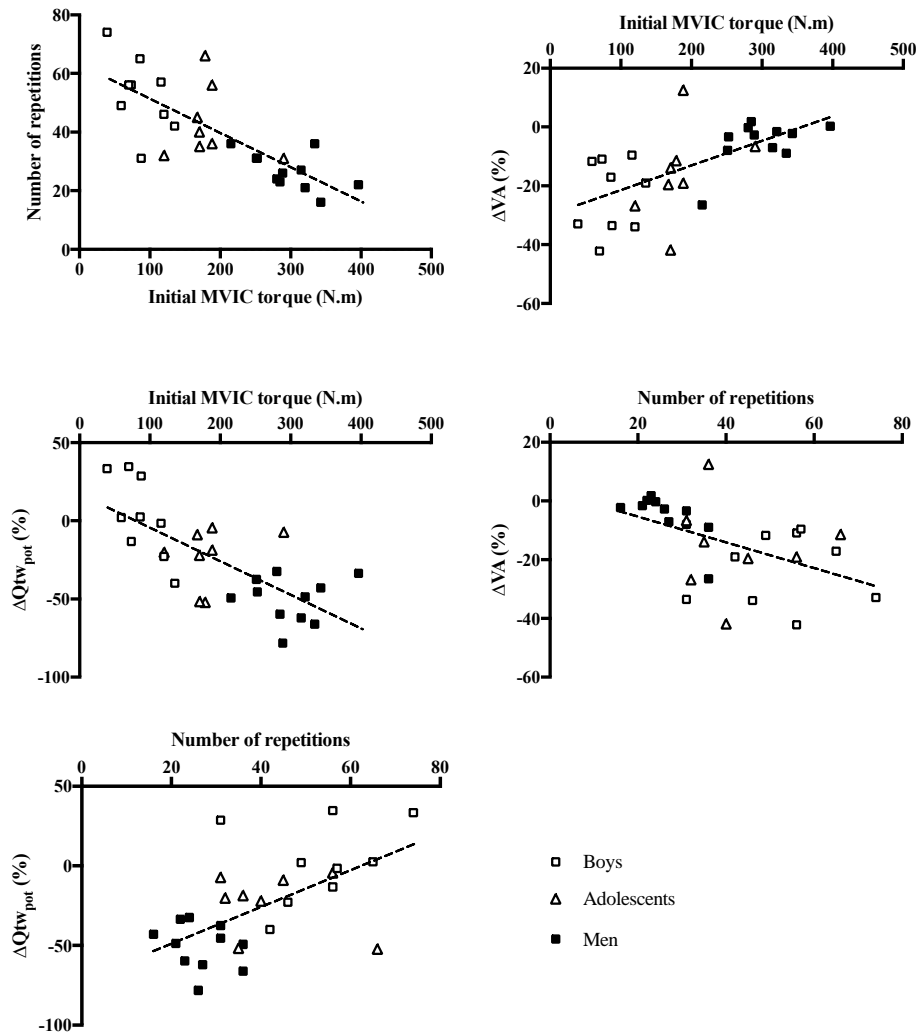

$$
\begin{aligned}
& \text { - Boys } \\
& \Delta \quad \text { Adolescents } \\
& \text { - Men }
\end{aligned}
$$


Fig. 4: Correlations between initial maximal voluntary isometric contraction (MVIC) torque, number of repetitions, change in potentiated twitch torque (Qtwpot) and change in voluntary activation level (VA). Boys, adolescents and men were pooled in the analysis.

\section{DISCUSSION}

The purpose of the present study was to investigate the effect of maturation on the development and etiology of neuromuscular fatigue induced by repeated MVIC of the KE muscles. The main results showed that adolescents tended to fatigue faster than prepubertal boys but slower than men during repeated maximal voluntary contractions. The greater fatigability in adolescents than prepubertal boys was associated with a greater peripheral fatigue and a similar central fatigue. In addition, the lower fatigability in adolescents than adults was associated with a lesser peripheral fatigue and a greater central fatigue. Finally, when MVIC was used as co-variable in the statistical analysis, differences of peripheral fatigue between groups persisted, suggesting that factors other than initial MVIC torque may account for the differences of peripheral fatigue during maturation.

The results of the present study confirm that adolescents fatigue faster than prepubertal children and slower than adults during repeated isometric maximal voluntary contractions of the knee extensors. The number of repetitions to task failure tended to be lower in adolescents than prepubertal boys and significantly higher in adolescents than men. Our results agree with the data published by Ratel et al. (2002) showing a higher decline of peak power output in adolescents than children or in adults than adolescents during ten repeated 10-s cycling sprints separated by $30 \mathrm{~s}$ of passive recovery. Similarly, it has been reported that during 4 series of 18 concentric knee flexions and extensions, the decline of peak torque is lower in prepubertal boys than male adolescents and higher in men than adolescents (Zafeiridis et al. 2005; Dipla et al. 2009). Finally, during 5 series of 6 maximal eccentric contractions of the elbow flexors, the decline of concentric maximal torque was found to be higher in adolescents than children and higher in adults than adolescents (Chen et al. 2014).

Although scientific evidence supports a higher fatigability in adolescents than children and a lower fatigability in adolescents than adults during high-intensity intermittent exercise, the mechanisms explaining this phenomenon still remain unclear.

\section{Peripheral mechanisms}

In prepubertal boys, no significant alteration of Qtw pot was observed during the fatigue protocol. In contrast, in adolescents and men, Qtwpot decreased significantly throughout the fatigue protocol and the decrement was significantly higher in men than adolescents. This suggests that contractile properties and/or excitation-contraction coupling were preserved in prepubertal boys and more strongly altered in men than adolescents. In contrast, the time-course of M-wave did not differ between groups, suggesting that no maturation-related change in the excitability of the sarcolemma was associated with fatigue. These results agree with previous studies, showing a lower alteration of the potentiated twitch torque during fatigue in prepubertal children than adults (Gorianovas et al. 2013; Murphy et al. 2014; Hatzikotoulas et al. 2014; Ratel et al. 2015; Piponnier et al. 2018). Furthermore, they concur with those published by Streckis et al. (2007) showing a lower alteration of twitch torque during a sustained 2-min maximal voluntary contraction of the knee extensors in 13.6-year-old girls and 13.9-year-old boys than adults. However, the results of the present study reveal for the first time an effect of maturation.

\section{Central mechanisms}

Regarding neural factors, prepubertal boys and male adolescents showed a significant VA and RMS.Mmax-1 (only in boys) decrement during the fatigue test, whereas no change of VA and RMS.Mmax-1 was observed in men. Moreover, the decrement of VA was similar in prepubertal 
boys and male adolescents. The interplay of central and peripheral mechanisms of fatigue through maturation remains to be elucidated; however, on the basis of these results, it could be suggested that the greater central fatigue in children and adolescents accounted for their lower peripheral fatigue than adults. Indeed, according to the central governor theory, the central nervous system could limit the recruitment of motor units to prevent any extensive homeostasis disturbance, muscle damage, and biological harm (Noakes et al. 2005). As such, some studies proposed that the central nervous system may "tolerate" a given peripheral fatigue level (Amann and Dempsey 2008; Millet 2011; Zghal et al. 2015). It is currently unknown if this tolerance is different in children, adolescents and adults, but the lower peripheral and the higher central fatigue reported here in children and adolescents suggest that this tolerance could be centrally set at a lower level than adults. Furthermore, the greater peripheral fatigue found for a comparable amount of central fatigue in adolescents than children suggests that the tolerance could be set at higher level in adolescents. This finding could support the idea that (i) the central nervous system could not tolerate the development of an extensive peripheral fatigue in children (Piponnier et al. 2018), contrary to adults, and (ii) the tolerance of the central nervous system to peripheral fatigue could increase during puberty.

Regarding to the central regulation of the antagonist coactivation, the results of the present study showed different patterns between groups, since it decreased in prepubertal boys whereas it remained unchanged in male adolescents and men. The progressive decrease of the coactivation level in prepubertal boys may have contributed to limit the loss of torque, and therefore to delay the level of fatigue, as previously reported (Piponnier et al. 2018). This potential mechanism could withdraw through maturation since no significant change of the antagonist coactivation during the fatigue protocol was observed in adolescents. However, these results should be confirmed since, to our knowledge, no other studies have investigated the effect of maturation on the antagonist coactivation associated with fatigue. Furthermore, other studies have reported opposite results when comparing prepubertal children and adults (Paraschos et al. 2007; Armatas et al. 2010; Murphy et al. 2014). Therefore, further studies are required to clarify this issue.

\section{Factors underpinning differences in neuromuscular fatigue}

Among the factors that may account for the differences of peripheral fatigue between prepubertal boys, male adolescents and men, is the absolute torque level. Indeed, when the three groups were pooled in the analysis, the first MVIC torque of the fatigue test was correlated to the twitch torque decrement. This suggestion is consistent with other studies that showed that the greater fatigue observed in men $v s$. women was eliminated if subjects were matched for absolute strength (Hunter et al. 2004). However, other factors may account for the differences of peripheral fatigue between prepubertal children, adolescents and adults. Indeed, the differential time-course of the potentiated twitch torque over repeated maximal voluntary contractions persisted between groups when the initial MVIC torque was used as co-variable. This result is consistent with recent reports (Piponnier et al. in press), showing that difference in torque level could not fully account for difference in neuromuscular fatigue at different muscle-tendon lengths between prepubertal children and adults. Thus, other factors could be involved in the development and etiology of neuromuscular fatigue during growth and maturation, such as the metabolic profile (Bontemps et al. 2019). Specifically, the greater proportion of slow-twitch fibers (Lexell et al. 1992) and the greater reliance on oxidative metabolism (Tonson et al. 2010) in prepubertal children may also could account for their reduced peripheral fatigue.

The higher implication of central factors in the development of fatigue in children and adolescents could also be ascribed to their lower absolute MVIC torque and as a result, to the concurrent longer exercise duration than adults. Indeed, in the present study, the number of 
repetitions and the associated exercise duration were clearly higher in prepubertal boys and male adolescents than adults. Furthermore, the relative VA loss was correlated to the first MVIC of the fatigue test and to the number of repetitions. These findings are consistent with another study (Thomas et al. 2015), reporting that central fatigue increases with exercise duration. Also, the results of the present study reveal no significant difference in the time-course of VA between groups when the MVIC torque was used as co-variable. These results agree with those published by Russ (2009), which reported similar changes in MVIC decrements and central activation in strength-matched men and women.

\section{CONCLUSIONS}

The results of the present study show that male adolescents fatigue faster than prepubertal children but slower than men during repeated maximal voluntary isometric contractions of the knee extensors. Furthermore, the contribution of central and peripheral mechanisms to neuromuscular fatigue differs between groups. Children and adolescents experience less peripheral and more central fatigue than adults. Moreover, adolescents experience more peripheral fatigue than children for a comparable amount of central fatigue. This could support the idea that (i) the central nervous system could not tolerate the development of an extensive peripheral fatigue in children, contrary to adults, and (ii) the tolerance of the central nervous system to peripheral fatigue could increase throughout maturation. However, further studies are required to better understand the origin of these central regulations and the interplay between peripheral and central mechanisms of fatigue throughout maturation.

\section{6}

467

468

\section{ACKNOWLEDGEMENTS}

Virginie Kluka was supported by a grant of the French National Agency of Technological Research (ANRT), n²012/0284.

\section{CONFLICT OF INTEREST}

The authors report no conflict of interest. A funding from the French National Agency of Technological Research (ANRT: ${ }^{\circ} 2012 / 0284$; Virginie Kluka) was received for this project. This work is known to and agreed by the co-authors identified on the manuscript's title page. This work required more than six people, because of clinical examination (physician or pediatrician), recruitment of volunteers, experimental procedures, statistical analysis and data analysis.

\section{REFERENCES}

Amann M, Dempsey JA (2008) Locomotor muscle fatigue modifies central motor drive in healthy humans and imposes a limitation to exercise performance. J Physiol 586:161-173. doi: 10.1113/jphysiol.2007.141838

Armatas V, Bassa E, Patikas D, et al (2010) Neuromuscular differences between men and prepubescent boys during a peak isometric knee extension intermittent fatigue test. Pediatr Exerc Sci 22:205-217

Bigland-Ritchie B, Woods JJ (1984) Changes in muscle contractile properties and neural control during human muscular fatigue. Muscle Nerve 7:691-699. doi: 10.1002/mus.880070902

Bontemps B, Piponnier E, Chalchat E, et al (2019) Children Exhibit a More Comparable Neuromuscular Fatigue Profile to Endurance Athletes Than Untrained Adults. Front Physiol 10:119. doi: 10.3389/fphys.2019.00119 
Burke D (2002) Effects of activity on axonal excitability: implications for motor control studies. Adv Exp Med Biol 508:33-37

Chen TC, Chen H-L, Liu Y-C, Nosaka K (2014) Eccentric exercise-induced muscle damage of pre-adolescent and adolescent boys in comparison to young men. Eur J Appl Physiol 114:11831195. doi: 10.1007/s00421-014-2848-3

Cohen J (1969) Statistical power analysis for Behavioral sciences. Academic Press.

Dipla K, Tsirini T, Zafeiridis A, et al (2009) Fatigue resistance during high-intensity intermittent exercise from childhood to adulthood in males and females. Eur J Appl Physiol 106:645-653. doi: 10.1007/s00421-009-1058-x

Enoka RM, Stuart DG (1992) Neurobiology of muscle fatigue. J Appl Physiol 72:1631-1648. doi: 10.1152/jappl.1992.72.5.1631

Gandevia SC (2001) Spinal and supraspinal factors in human muscle fatigue. Physiol Rev 81:1725-1789. doi: 10.1152/physrev.2001.81.4.1725

Garrandes F, Colson SS, Pensini M, et al (2007) Neuromuscular fatigue profile in endurancetrained and power-trained athletes. Med Sci Sports Exerc 39:149-158. doi: 10.1249/01.mss.0000240322.00782.c9

Gorianovas G, Skurvydas A, Streckis V, et al (2013) Repeated bout effect was more expressed in young adult males than in elderly males and boys. BioMed Res Int 2013:218970. doi: $10.1155 / 2013 / 218970$

Hamada T, Sale DG, MacDougall JD, Tarnopolsky MA (2003) Interaction of fibre type, potentiation and fatigue in human knee extensor muscles. Acta Physiol Scand 178:165-173. doi: 10.1046/j.1365-201X.2003.01121.x

Hatzikotoulas K, Patikas D, Ratel S, et al (2014) Central and peripheral fatigability in boys and men during maximal contraction. Med Sci Sports Exerc 46:1326-1333. doi: 10.1249/MSS.0000000000000239

Hermens HJ, Freriks B, Disselhorst-Klug C, Rau G (2000) Development of recommendations for SEMG sensors and sensor placement procedures. J Electromyogr Kinesiol 10:361-374

Hunter SK, Critchlow A, Shin I-S, Enoka RM (2004) Fatigability of the elbow flexor muscles for a sustained submaximal contraction is similar in men and women matched for strength. $\mathrm{J}$ Appl Physiol 96:195-202. doi: 10.1152/japplphysiol.00893.2003

Kent-Braun JA, Ng AV, Doyle JW, Towse TF (2002) Human skeletal muscle responses vary with age and gender during fatigue due to incremental isometric exercise. J Appl Physiol 93:1813-1823. doi: 10.1152/japplphysiol.00091.2002

Lexell J, Sjöström M, Nordlund AS, Taylor CC (1992) Growth and development of human muscle: a quantitative morphological study of whole vastus lateralis from childhood to adult age. Muscle Nerve 15:404-409. doi: 10.1002/mus.880150323

Merton PA (1954) Voluntary strength and fatigue. J Physiol 123:553-564

Millet GY (2011) Can neuromuscular fatigue explain running strategies and performance in ultra-marathons?: the flush model. Sports Med Auckl NZ 41:489-506. doi: 10.2165/11588760000000000-00000

Mira J, Aboodarda SJ, Floreani M, et al (2018) Effects of endurance training on neuromuscular fatigue in healthy active men. Part I: Strength loss and muscle fatigue. Eur J Appl Physiol. doi: 10.1007/s00421-018-3950-8 
Mirwald RL, Baxter-Jones ADG, Bailey DA, Beunen GP (2002) An assessment of maturity

533 from anthropometric measurements. Med Sci Sports Exerc 34:689-694

534 Murphy JR, Button DC, Chaouachi A, Behm DG (2014) Prepubescent males are less 535 susceptible to neuromuscular fatigue following resistance exercise. Eur J Appl Physiol 536 114:825-835. doi: 10.1007/s00421-013-2809-2

537

538

539

540

541

542

543

544

545

546

547

548

549

550

551

552

553

554

555

556

557

558

559

560

561

562

563

564

565

566

567

568

569

570

571

572

573

574

Neyroud D, Vallotton A, Millet GY, et al (2014) The effect of muscle fatigue on stimulus intensity requirements for central and peripheral fatigue quantification. Eur J Appl Physiol 114:205-215. doi: 10.1007/s00421-013-2760-2

Noakes TD, St Clair Gibson A, Lambert EV (2005) From catastrophe to complexity: a novel model of integrative central neural regulation of effort and fatigue during exercise in humans: summary and conclusions. Br J Sports Med 39:120-124. doi: 10.1136/bjsm.2003.010330

Paraschos I, Hassani A, Bassa E, et al (2007) Fatigue differences between adults and prepubertal males. Int J Sports Med 28:958-963. doi: 10.1055/s-2007-964984

Piponnier E, Martin V, Bontemps B, et al (2018) Child-adult differences in neuromuscular fatigue are muscle-dependent. J Appl Physiol 1246-1256. doi: 10.1152/japplphysiol.00244.2018

Piponnier E, Martin V, Chalchat E, et al (in press) Effect of MTU length on child-adult difference in neuromuscular fatigue. Med Sci Sports Exerc

Ratel S, Bedu M, Hennegrave A, et al (2002) Effects of age and recovery duration on peak power output during repeated cycling sprints. Int J Sports Med 23:397-402. doi: 10.1055/s2002-33737

Ratel S, Blazevich AJ (2017) Are Prepubertal Children Metabolically Comparable to WellTrained Adult Endurance Athletes? Sports Med Auckl NZ 47:1477-1485. doi: 10.1007/s40279-016-0671-1

Ratel S, Kluka V, Vicencio SG, et al (2015) Insights into the Mechanisms of Neuromuscular Fatigue in Boys and Men. Med Sci Sports Exerc 47:2319-2328. doi: 10.1249/MSS.0000000000000697

Russ D (2009) Sex differences in muscle fatigue. In: Williams C, Ratel S (eds) Human muscle fatigue. London and New-York

Souron R, Nosaka K, Jubeau M (2018) Changes in central and peripheral neuromuscular fatigue indices after concentric versus eccentric contractions of the knee extensors. Eur J Appl Physiol 118:805-816. doi: 10.1007/s00421-018-3816-0

Streckis V, Skurvydas A, Ratkevicius A (2007) Children are more susceptible to central fatigue than adults. Muscle Nerve 36:357-363. doi: 10.1002/mus.20816

Tanner JM, Whitehouse RH (1976) Clinical longitudinal standards for height, weight, height velocity, weight velocity, and stages of puberty. Arch Dis Child 51:170-179

Thomas K, Goodall S, Stone M, et al (2015) Central and peripheral fatigue in male cyclists after 4-, 20-, and 40-km time trials. Med Sci Sports Exerc 47:537-546. doi: 10.1249/MSS.0000000000000448

Tonson A, Ratel S, Le Fur Y, et al (2008) Effect of maturation on the relationship between muscle size and force production. Med Sci Sports Exerc 40:918-925. doi: 10.1249/MSS.0b013e3181641bed

Tonson A, Ratel S, Le Fur Y, et al (2010) Muscle energetics changes throughout maturation: a 
575 quantitative 31P-MRS analysis. J Appl Physiol 109:1769-1778. doi: 576 10.1152/japplphysiol.01423.2009

577 Zafeiridis A, Dalamitros A, Dipla K, et al (2005) Recovery during high-intensity intermittent 578 anaerobic exercise in boys, teens, and men. Med Sci Sports Exerc 37:505-512

579 Zghal F, Cottin F, Kenoun I, et al (2015) Improved tolerance of peripheral fatigue by the central 580 nervous system after endurance training. Eur J Appl Physiol 115:1401-1415. doi: $581 \quad 10.1007 / \mathrm{s} 00421-015-3123-y$ 